\title{
A Low-Complexity Demodulation for Oversampled LoRa Signal
}

\author{
Vincent Savaux
}

\begin{abstract}
This paper deals with a method of demodulation for oversampled LoRa signal. The usual maximum likelihood (ML) based demodulation method for LoRa chirp spread spectrum (CSS) waveform is dedicated to signals sampled at Nyquist rate, whereas considering oversampled signals may improve the performance of the LoRa demodulation process. In this respect, when an oversampling rate (OSR) 2 is assumed, the method suggested in this paper consists in applying two demodulation processes to the even and odd samples of the oversampled LoRa signal, and then combining the results. This principle is then generalized to any OSR, and we show that the complexity of the method is low since it only involves discrete Fourier transforms (DFT). Moreover, a performance analysis in terms of symbol and bit error rate (SER and BER) is presented considering both additive white Gaussian noise (AWGN) and Rayleigh channel models. Simulations show the relevance of the method and the performance analysis as a gain of $3 \mathrm{~dB}$ is achieved by the demodulation at OSR 2 compared with OSR 1.
\end{abstract}

Keywords - LoRa, Demodulation, Maximum Likelihood, Low-complexity

\section{INTRODUCTION}

In the recent years, the Internet of things (IoT) technologies, among which the low power wide area network (LPWA) solutions, have enabled the connectivity of a constantly growing number of devices, offering digital transformation across industry verticals [1]. Among the LPWA technologies, LoRa is one of the most deployed and studied by both academic and industrial researchers, mainly because it offers the advantage of an easy deployment. The LoRa waveform is originally based on the chirp spread spectrum (CSS) modulation, described by Winkler in [2]. More generally, the physical layer (PHY) of LoRa is developed by Semtech, and the system has been promoted by the LoRa Alliance, that specifies the LoRaWAN open protocol.

The PHY layer of LoRa as well as related signal processing algorithms and performance analyzes have been extensively studied in the literature. Thus, the LoRa waveform and its main properties are described by the authors of [3], [4]. In [5]-[9], theoretical symbol and bit error rate (SER and BER) expressions and approximations are provided for different channel models. However, these performance analyzes are limited to LoRa signals sampled at Nyquist rate, because the LoRa demodulation is inherently designed to process signals sampled at oversampling rate (OSR) 1 (i.e. Nyquist rate). For this reason, only few papers like [10] deal with demodulation of oversampled 
LoRa signals, whereas it is a straightforward strategy for performance improvement. In [10] a discrete Fourier transform (DFT) of the size of the oversampled signal is applied, and the resulting spectrum is processed to take advantage of the gain of energy brought by the oversampling. Despite this method requires a low computational cost, the processing in the frequency domain leads to an increase of the observed noise level, making the technique relevant only for OSR strictly larger than 2 .

In this paper, we suggest a new demodulation method for oversampled LoRa signals combining both good performance and low complexity. The principle of the method considering OSR 2, consists in splitting the oversampled signal into two signals sampled at Nyquist rate, one containing the even samples, and the other one containing the odd samples of the oversampled LoRa signal. Then, dedicated DFT are applied to each of the signals, and the resulting responses are combined to apply the usual LoRa demodulation process. It is shown that such principle can be extended to any OSR as well. The complexity of the suggested technique is limited as it only involves to apply a number of DFT equal to the OSR. Furthermore, the theoretical achievable SER and BER performance is derived considering both additive white Gaussian noise (AWGN) and Rayleigh channel models, and it is validated through simulations. Thus, a gain of $3 \mathrm{~dB}$ is achieved by the suggested demodulation at OSR 2 compared with the usual demodulation at Nyquist rate.

The rest of the paper is organized as follows: Section II presents a background on LoRa modulation and demodulation. In Section III we introduce the new demodulation method for oversampled LoRa signal, as well as the corresponding performance analysis. Simulations results show the relevance of the technique in Section IV, and Section V concludes this paper.

Notations: The normal font $x$ is used for scalars, and the boldface $\mathbf{x}$ for vectors and matrices. The lower-case and the upper-case $x$ and $X$ indicates samples in time and frequency domains, respectively. The probability of an event $E$ is denoted by $\mathbb{P}(E)$, and $\mathbb{E}\{$.$\} is the mathematical expectation.$

\section{LORA SySTEM ModeL}

In this section, we describe the LoRa signal model and the corresponding demodulation et the receiver side. Based on CSS, the modulation of LoRa signal consists in mapping a binary packet of size $N=2^{S F}$, where $S F$ is the spreading factor, onto a LoRa symbol noted $s^{(m)}$, where $m=0,1, . ., N-1$ indicates the index of the modulated chirp. Each symbol has a duration $T_{s}$ and a bandwidth $B_{w}$ such that $B_{w} T_{s}=N$. We denote by $r_{o}$ the OSR of the signal ( $r=1$ means Nyquist rate), then for any $n=0,1, . ., N^{\prime}-1$ where $N^{\prime}=r N$, and for any $S F$ value, the modulated LoRa symbol $s^{(m)}$ can be expressed by generalizing [4]-(13) as

$$
s^{(m)}[n]=\exp \left(2 j \pi \frac{n}{r_{o}}\left(\frac{n}{2 N^{\prime}}-\frac{1}{2}+\frac{m}{N}-u\left(\frac{n}{r_{o}}-N+m\right)\right)\right),
$$

where $u($.$) indicates the Heaviside step function. We assume a synchronized reception (see [11]-[14] for synchro-$ nization in LoRa), and we consider that the propagation channel can be modeled as a one-tap block fading channel $h$, therefore, for any $n=0,1, . . N^{\prime}-1$, the received signal $r[n]$ is given by 


$$
r[n]=h s^{(m)}[n]+w[n],
$$

where $w[n], n=0,1, . ., N^{\prime}-1$, are the independent and identically distributed (iid) samples of the complex additive white Gaussian noise (AWGN) such that $w[n] \sim \mathbb{C N}\left(0, \sigma^{2}\right)$, where $\sigma^{2}=\mathbb{E}\left\{|w[n]|^{2}\right\}$. In the following, we assume that channel $h$ is either an AWGN channel (i.e. $h=1$ ) or a Rayleigh channel (i.e. $h=\sim \mathbb{C N}\left(0, \sigma_{h}^{2}\right)$ ). In any case, we define the signal-to-noise ratio as

$$
S N R=\frac{\mathbb{E}\left\{\left|h s^{(m)}[n]\right|^{2}\right\}}{\mathbb{E}\left\{|w[n]|^{2}\right\}}=\frac{\mathbb{E}\left\{|h|^{2}\right\}}{\sigma^{2}} .
$$

The basic principle of the LoRa demodulation process consists in estimating the symbol index $m$ from the observation $r[n]$, by means of the maximum likelihood (ML) estimator since it is optimal in condition of synchronized reception [5], [12], [13]. For OSR $r_{o}=1$, the LoRa demodulation process is based on three mains steps: the "dechirp" operation, consisting in removing the quadratic component from the received LoRa symbol:

$$
y[n]=r[n] \exp \left(-2 j \pi n\left(\frac{n}{2 N}-\frac{1}{2}\right)\right) .
$$

Then, a discrete Fourier transform (DFT) is applied:

$$
Y[k]=\frac{1}{\sqrt{N}} \sum_{n=0}^{N-1} y[n] e^{\frac{-2 j \pi n k}{N}},
$$

where $k=0,1, . ., N-1$. It should be noticed that $Y[k]=\sqrt{N} \delta(k-m)+W[k]$ when $k=m$, and $Y[k]=W[k]$ when $k \neq m$, where $\delta(k)$ is the Dirac impulse and $W[k]$ are the noise samples in frequency domain. Moreover, by property of the DFT, the noise samples $W[k]$ are all iid and $W[k] \sim \mathbb{C N}\left(0, \sigma^{2}\right)$. Finally, the ML estimator of $m$ yields

$$
\hat{m}=\underset{k \in \llbracket 0, N \rrbracket}{\arg \max }|Y[k]|^{2},
$$

where $|Y[k]|^{2}$ is called the periodogram of $y[n]$. The symbol error rate (SER) performance corresponding to the LoRa demodulation has been largely studied in from [6]-[8]. Thus, given the channel gain $h$, it can be expressed as

$$
\begin{aligned}
P_{s \mid h} & =\mathbb{P}(\hat{m} \neq m \mid h) \\
& =-\sum_{k=1}^{N-1} \frac{(-1)^{k}\left(\begin{array}{c}
N-1 \\
k
\end{array}\right)}{k+1} \exp \left(-\frac{k N|h|^{2}}{(k+1) \sigma^{2}}\right) .
\end{aligned}
$$

From (8), the SER performance of the LoRa signal in AWGN channel is simply obtained through $P_{s}^{a w g n}=P_{s \mid h=1}$, and the SER of the LoRa signal over Rayleigh channel, denoted by $P_{s}^{r a y}$, is given by averaging $P_{s \mid h}$ weighted by the Rayleigh distribution $f_{|h|^{2}}(x)=\frac{e^{-\frac{x}{\sigma_{h}^{2}}}}{\sigma_{h}^{2}}$ such as follows 


$$
\begin{aligned}
P_{s}^{r a y} & =\int_{0}^{+\infty} f_{|h|^{2}}(x) P_{s \mid h} d x \\
& =-\sum_{k=1}^{N-1} \frac{(-1)^{k}\left(\begin{array}{c}
N-1 \\
k
\end{array}\right) \sigma^{2}}{k N \sigma_{h}^{2}+(k+1) \sigma^{2}} .
\end{aligned}
$$

The BER $P_{b}$ is, in turn, obtained from the SER through $P_{b}=\frac{2^{S F-1}}{2^{S F}-1} P_{s}$. Besides the performance analysis, we also gives the complexity of the LoRa demodulation in terms of complex multiplications: we deduce from (4), (5), and (6) that these operations require $N, N \log (N)$, and $N$ operations, respectively.

In [10], the demodulation of LoRa has been extended to any OSR $r_{o}>1$. Similar to the case OSR $r_{o}=1$, the "dechirp" operation becomes

$$
y[n]=r[n] \exp \left(-2 j \pi \frac{n}{r_{o}}\left(\frac{n}{2 N^{\prime}}-\frac{1}{2}\right)\right)
$$

where $n=0,1, . ., N^{\prime}-1$. A DFT of size $N^{\prime}$ is then applied to $y[n]$ in (11). The resulting spectrum $Y[k]$, $k=0,1, \ldots, N^{\prime}-1$, highlights two peaks at the positions $k=m$ and $k=m-N+N^{\prime}$, and with amplitudes $Y[k=m]=\frac{r_{o}(N-m)}{\sqrt{N^{\prime}}} \delta(k-m)+W[m]$ and $Y\left[k=m-N+N^{\prime}\right]=\frac{r_{o} m}{\sqrt{N^{\prime}}} \delta\left(k-m+N-N^{\prime}\right)+W\left[m-N+N^{\prime}\right]$. As a consequence, it is suggested in [10] to sum these peaks through

$$
\tilde{Y}[k]=Y[k]+Y\left[\left(r_{o}-1\right) N+k\right] .
$$

It must be noticed that $\tilde{Y}[k]=\sqrt{N^{\prime}} \delta(k-m)+\tilde{W}[k]$ when $k=m$, and $\tilde{Y}[k]=\tilde{W}[k]$ when $k \neq m$, where $\tilde{W}[k]=W[k]+W\left[\left(r_{o}-1\right) N+k\right]$ are the combined noise samples in frequency domain. Finally, the ML estimators of the index $m$ then leads to

$$
\hat{m}=\underset{k \in \llbracket 0, N \rrbracket}{\arg \max }|\tilde{Y}[k]|^{2} .
$$

The SER performance of the LoRa demodulation proposed in [10], for any OSR $r>1$ is given by

$$
P_{s \mid h}=-\sum_{k=1}^{N-1} \frac{(-1)^{k}\left(\begin{array}{c}
N-1 \\
k
\end{array}\right)}{k+1} \exp \left(-\frac{k N^{\prime}|h|^{2}}{2(k+1) \sigma^{2}}\right),
$$

where the term $2 \sigma^{2}$ in the denominator within the exponential comes from $\mathbb{E}\left\{\left|W[k]+W\left[\left(r_{o}-1\right) N+k\right]\right|^{2}\right\}=2 \sigma^{2}$. It can be deduced from (14) that an OSR $r_{o}=2$ leads to the same SER as in (8) using $r_{o}=1$, and then a performance improvement of the method in [10] is only effective for $r_{o}>2$ compared with the usual LoRa demodulation at OSR $r_{o}=1$. More generally, the larger $N^{\prime}$ (through the OSR $r_{o}$ ), the lower the SER, but to the cost of increase of complexity. In the following, we introduce a new low-complex LoRa demodulation method that outperforms the usual demodulation for any OSR $r_{o} \geq 2$. 


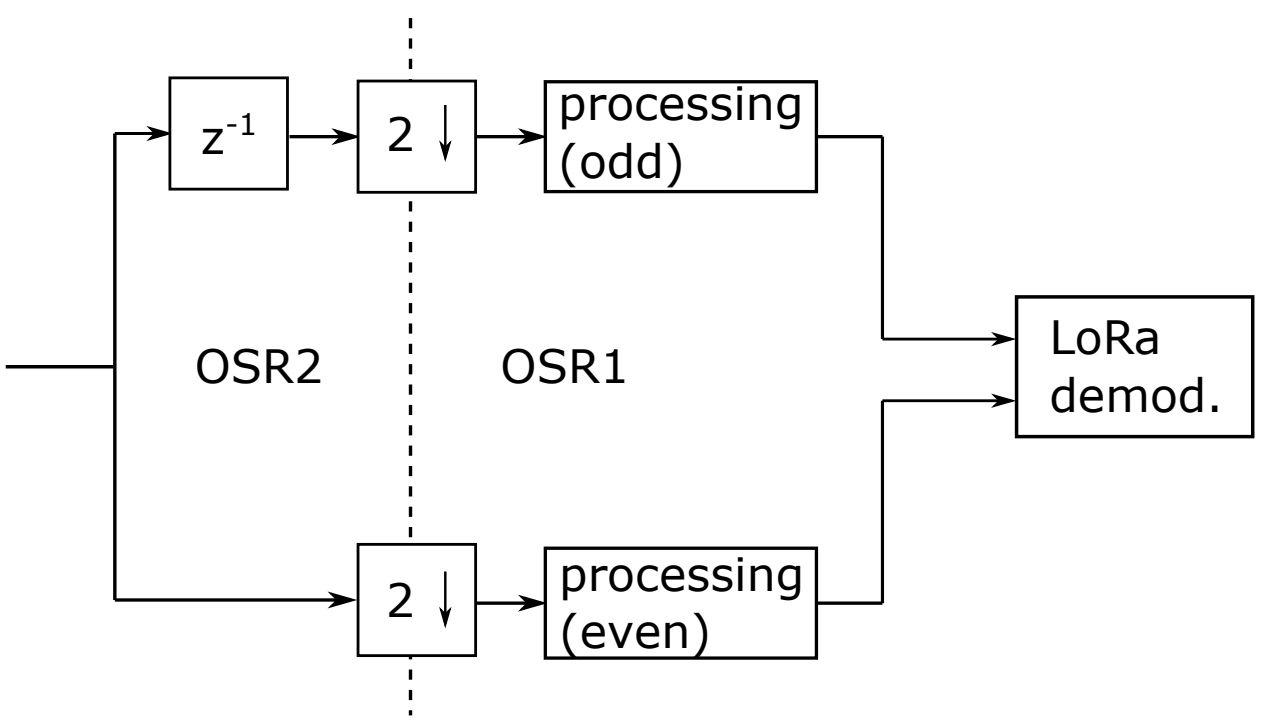

Fig. 1. Principle of the suggested demodulation for LoRa signal at OSR $r=2$.

\section{NEW DEMOdulation OF OVERSAMPLED LORA SignAL}

A. $O S R r_{o}=2$

We first present the proposed LoRa demodulation method for OSR $r_{o}=2$ before generalizing to any OSR. The principle is illustrated in Fig. 1: the received signal $r[n]$ sampled at OSR $r_{o}=2$ is split into two downsampled signals, one consisting in the even samples and the other one consisting in the odd samples (illustrated by the delay $z^{-1}$ in Fig. 1) of the received oversampled signal. Then, specific "dechirp" and DFT processes are applied, and the resulting signals are combined to apply the usual LoRa demodulation (i.e. the ML estimator of $m$ ). In the following, the steps of the suggested LoRa demodulation are detailed.

1) $\operatorname{OSR} r_{o}=2$, $n$ Even: For $n$ even, we note $n=2 p$, with $p=0,1, . ., N-1$. In that case, the ML estimator of $m$ applied to the observations $r[2 p]$ reduces to the aforementioned case corresponding to OSR 1 , such as shown as follows:

$$
\begin{aligned}
\hat{m} & =\underset{k \in \llbracket 0, N \rrbracket}{\arg \max }\left|\frac{1}{\sqrt{N}} \sum_{p=0}^{N-1} r[2 p] s^{(k)}[2 p]^{*}\right|^{2} \\
& =\underset{k \in \llbracket 0, N \rrbracket}{\arg \max }\left|\frac{1}{\sqrt{N}} \sum_{p=0}^{N-1} r[2 p] \exp \left(-2 j \pi p\left(\frac{p}{N^{\prime}}-\frac{1}{2}+\frac{k}{N}-u(p-N+k)\right)\right)\right|^{2},
\end{aligned}
$$

and since $\exp (2 j \pi p u(p-N+k))=1$ for any $p$ and $k$ values, we obtain:

$$
\begin{aligned}
\hat{m} & =\underset{k \in \llbracket 0, N \rrbracket}{\arg \max }\left|\frac{1}{\sqrt{N}} \sum_{p=0}^{N-1} r[2 p] s^{(0)}[2 p]^{*} \exp \left(-2 j \pi \frac{p k}{N}\right)\right|^{2} \\
& =\underset{k \in \llbracket 0, N \rrbracket}{\arg \max }|Y[k]|^{2} .
\end{aligned}
$$


2) $O S R r_{o}=2, n$ Odd: For $n$ odd, we note $n=2 p+1$, with $p=0,1, . ., N-1$. In that case, the ML estimator of $m$ applied to the observation $r[2 p+1]$ yields:

$$
\begin{aligned}
\hat{m} & =\underset{k \in \llbracket 0, N \rrbracket}{\arg \max }\left|\frac{1}{\sqrt{N}} \sum_{p=0}^{N-1} r[2 p+1] s^{(k)}[2 p+1]^{*}\right|^{2} \\
& =\underset{k \in \llbracket 0, N \rrbracket}{\arg \max }\left|\frac{1}{\sqrt{N}} \sum_{p=0}^{N-1} r[2 p+1] \exp \left(-2 j \pi \frac{2 p+1}{2}\left(\frac{2 p+1}{2 N^{\prime}}-\frac{1}{2}+\frac{k}{N}-u\left(\frac{2 p+1}{2}-N+k\right)\right)\right)\right|^{2} .
\end{aligned}
$$

Then, since $\exp \left(2 j \pi p u\left(\frac{2 p+1}{2}-N+k\right)\right)=1$ for any $p$ and $k$ values, and $\left|\exp \left(-j \pi \frac{k}{N}\right)\right|=1$ we obtain:

$$
\begin{aligned}
\hat{m} & =\underset{k \in \llbracket 0, N \rrbracket}{\arg \max }\left|\frac{1}{\sqrt{N}} \sum_{p=0}^{N-1} r[2 p+1] s^{(0)}[2 p+1]^{*} \exp \left(-j \pi \frac{k}{N}\right) \exp \left(j \pi u\left(\frac{2 p+1}{2}-N+k\right)\right) \exp \left(-2 j \pi \frac{p k}{N}\right)\right|^{2}(21) \\
& =\underset{k \in \llbracket 0, N \rrbracket}{\arg \max }\left|\frac{\exp \left(-j \pi \frac{k}{N}\right)}{\sqrt{N}} \sum_{p=0}^{N-1} r[2 p+1] s^{(0)}[2 p+1]^{*} \exp \left(j \pi u\left(\frac{2 p+1}{2}-N+k\right)\right) \exp \left(-2 j \pi \frac{p k}{N}\right)\right|^{2} \\
& =\underset{k \in \llbracket 0, N \rrbracket}{\arg \max } \mid Y^{\prime}[k]^{2}
\end{aligned}
$$

where $Y^{\prime}[k]$ is the DFT of $r[2 p+1] s^{(0)}[2 p+1]^{*} \exp \left(j \pi u\left(\frac{2 p+1}{2}-N+k\right)\right)$. Alternatively, we can rewrite $Y^{\prime}[k]$ in (23) by noticing that

$$
\exp \left(j \pi u\left(\frac{2 p+1}{2}-N+k\right)\right)=\left\{\begin{array}{l}
1, \text { if } \frac{2 p+1}{2}-N+k<0 \\
-1, \text { if } \frac{2 p+1}{2}-N+k \geq 0
\end{array} .\right.
$$

Then $Y^{\prime}[k]$ in (23) can be expressed in a matrix notation as

$$
\mathbf{Y}^{\prime}=\underbrace{\left(\begin{array}{cccccc}
1 & 1 & 1 & \cdots & 1 & 1 \\
1 & \omega & \omega^{2} & \cdots & \omega^{N-2} & -\omega^{N-1} \\
1 & \omega^{2} & \omega^{4} & \cdots & -\omega^{2(N-2)} & -\omega^{2(N-1)} \\
\vdots & \vdots & \vdots & \ddots & \vdots & \vdots \\
1 & -\omega^{N-1} & -\omega^{2(N-1)} & \cdots & & -\omega^{(N-1)^{2}}
\end{array}\right)}_{\mathbf{F}}
$$

where $\mathbf{Y}^{\prime}$ and $\mathbf{y}$ are the $N \times 1$ vectors containing the samples $Y^{\prime}[k]$ and $r[2 p+1] s^{(0)}[2 p+1]^{*}$, respectively, and $\omega=e^{-2 j \frac{\pi}{N}}$. Furthermore, it must be emphasized that $\mathbf{F}^{\prime}$ is similar to the DFT matrix, as it only differs from the usual DFT matrix by signs. Thus (25) can be computed with the same complexity (in terms of complex multiplications) as the usual DFT, namely $N \log (N)$. 
3) $\operatorname{OSR} r_{o}=2$, Combining Even and Odd $n$ : Once both $Y[k]$ and $Y^{\prime}[k]$ have been obtain from the oversampled signal $y[n]$, it is possible to combine these results to improve the LoRa demodulation. To this end, the ML estimation of $m$ becomes:

$$
\hat{m}=\underset{k \in \llbracket 0, N \rrbracket}{\arg \max }|\underbrace{Y[k]+\exp \left(-j \pi \frac{k}{N}\right) Y^{\prime}[k]}_{\tilde{Y}[k]}|^{2} .
$$

Thus, it results that $\tilde{Y}[k]=2 \sqrt{N} \delta(k-m)+W[k]+e^{-j \pi \frac{k}{N}} W^{\prime}[k]$ when $k=m$, and $\tilde{Y}[k]=W[k]+e^{-j \pi \frac{k}{N}} W^{\prime}[k]$ when $k \neq m$. Since $\mathbb{E}\left\{\left|W[k]+e^{-j \pi \frac{k}{N}} W^{\prime}[k]\right|^{2}\right\}=2 \sigma^{2}$, the SER of the suggested demodulation leads to

$$
P_{s \mid h}=-\sum_{k=1}^{N-1} \frac{(-1)^{k}\left(\begin{array}{c}
N-1 \\
k
\end{array}\right)}{k+1} \exp \left(-\frac{2 k N|h|^{2}}{(k+1) \sigma^{2}}\right) .
$$

From (27) we notice that the ratio $\frac{2 k N|2|^{2}}{(k+1) \sigma^{2}}$ within the exponential is twice larger than the ratio $\frac{k N|2|^{2}}{(k+1) \sigma^{2}}$ in (8). Since the larger the ratio, the lower the SER, we deduce that the suggested method at OSR $r_{o}=2$ actually outperforms the usual LoRa demodulation at OSR $r_{o}=1$. Note that the SER in AWGN and Rayleigh channel can be straightforwardly obtained as previously, i.e. $P_{s}^{a w g n}=P_{s \mid h=1}$, and

$$
\begin{aligned}
P_{s}^{r a y} & =\int_{0}^{+\infty} f_{|h|^{2}}(x) P_{s \mid h} d x \\
& =-\sum_{k=1}^{N-1} \frac{(-1)^{k}\left(\begin{array}{c}
N-1 \\
k
\end{array}\right) \sigma^{2}}{2 k N \sigma_{h}^{2}+(k+1) \sigma^{2}} .
\end{aligned}
$$

Moreover the complexity of the suggested demodulation is only twice that of OSR $r_{o}=1$, since the processes for even and odd $n$ samples are similar.

\section{B. Generalizing for any OSR}

We can generalizing the principle of the demodulation described in Fig. 1 to any OSR. For any $r_{o} \geq 1$, the oversampled LoRa signal is split into $r_{o}$ branches, such that we denote $n=r_{o} p+q$ the indexes of the samples of the downsampled signal in the $q$-th branch, where $p=0,1, . ., N-1, q=0,1, r_{o}-1$. In that case, the ML estimator of $m$ applied to the observation $r\left[r_{o} p+q\right]$ yields:

$$
\begin{aligned}
\hat{m} & =\underset{k \in \llbracket 0, N \rrbracket}{\arg \max }\left|\frac{1}{\sqrt{N}} \sum_{p=0}^{N-1} r\left[r_{o} p+q\right] s^{(k)}\left[r_{o} p+q\right]^{*}\right|^{2} \\
& =\underset{k \in \llbracket 0, N \rrbracket}{\arg \max }\left|\frac{1}{\sqrt{N}} \sum_{p=0}^{N-1} r\left[r_{o} p+q\right] \exp \left(-2 j \pi \frac{r_{o} p+q}{r_{o}}\left(\frac{r_{o} p+q}{2 N^{\prime}}-\frac{1}{2}+\frac{k}{N}-u\left(\frac{r_{o} p+q}{r_{o}}-N+k\right)\right)\right)\right|^{2} .
\end{aligned}
$$

Then, since $\exp \left(2 j \pi p u\left(\frac{r_{o} p+1}{r_{o}}-N+k\right)\right)=1$ for any $p$ and $k$ values, and $\left|\exp \left(-2 j \pi \frac{q k}{r_{o} N}\right)\right|=1$ we obtain: 


$$
\begin{aligned}
\hat{m} & =\underset{k \in \llbracket 0, N \rrbracket}{\arg \max }\left|\frac{1}{\sqrt{N}} \sum_{p=0}^{N-1} r\left[r_{o} p+q\right] s^{(0)}\left[r_{o} p+q\right]^{*} \exp \left(-2 j \pi \frac{r_{o} p+q}{r_{o}}\left(\frac{k}{N}-u\left(\frac{r_{o} p+q}{r_{o}}-N+k\right)\right)\right)\right|^{2} \\
& =\underset{k \in \llbracket 0, N \rrbracket}{\arg \max } \mid \frac{\exp \left(-2 j \pi \frac{q k}{N r_{o}}\right)}{\sqrt{N}} \sum_{p=0}^{N-1} r\left[r_{o} p+q\right] s^{(0)}\left[r_{o} p+q\right]^{*} \exp \left(2 j \pi \frac{q}{r_{o}} u\left(\frac{r_{o} p+q}{r_{o}}-N+k\right)\right) \exp \left(-2 j \pi \frac{p k}{N}\right)\left(\beta 33^{2}\right) \\
& =\underset{k \in \llbracket 0, N \rrbracket}{\arg \max }\left|Y^{\left(r_{o}\right)}[k]\right|^{2}
\end{aligned}
$$

where $Y^{\left(q, r_{o}\right)}[k]$ is the DFT of $r\left[r_{o} p+q\right] s^{(0)}\left[r_{o} p+q\right]^{*} \exp \left(2 j \pi \frac{q}{r_{o}} u\left(\frac{r_{o} p+q}{r_{o}}-N+k\right)\right)$. Once again, we can rewrite (34) by noticing that

$$
\exp \left(2 j \pi \frac{q}{r_{o}} u\left(\frac{r_{o} p+q}{r_{o}}-N+k\right)\right)=\left\{\begin{array}{l}
1, \text { if } \frac{r_{o} p+q}{r_{o}}-N+k<0 \\
e^{2 j \pi \frac{q}{r_{o}}}, \text { if } \frac{r_{o} p+q}{r_{o}}-N+k \geq 0
\end{array} .\right.
$$

Then $Y^{\left(q, r_{o}\right)}[k]$ in (34) can be expressed in a matrix notation as

$$
\mathbf{Y}^{\left(q, r_{o}\right)}=\underbrace{\left(\begin{array}{cccccc}
1 & 1 & 1 & \cdots & 1 & 1 \\
1 & \omega & \omega^{2} & \cdots & \omega^{N-2} & e^{2 j \pi \frac{q}{r_{o}}} \omega^{N-1} \\
1 & \omega^{2} & \omega^{4} & \cdots & e^{2 j \pi \frac{q}{r_{o}}} \omega^{2(N-2)} & e^{2 j \pi \frac{q}{r_{o}}} \omega^{2(N-1)} \\
\vdots & \vdots & \vdots & \ddots & \vdots & \vdots \\
1 & e^{2 j \pi \frac{q}{r_{o}} \omega^{N-1}} & e^{2 j \pi \frac{q}{r_{o}}} \omega^{2(N-1)} & \cdots & & e^{2 j \pi \frac{q}{r_{o}}} \omega^{(N-1)^{2}}
\end{array}\right)}_{\mathbf{F}^{\left(q, r_{o}\right)}}
$$

where the complexity of the matrix multiplication straightforwardly reduces to that of the DFT if $r_{o} \in\{1,2,4\}$. For other values of $r_{o}$, dedicated algorithms for matrix multiplication may be necessary, which limits the ease of implementation. Then, similarly to (26) we can combine the results from the $r_{o}$ branches to apply the ML estimator as follows:

$$
\hat{m}=\underset{k \in \llbracket 0, N \rrbracket}{\arg \max }\left|\sum_{q=0}^{r_{o}-1} \exp \left(-2 j \pi \frac{q k}{N r_{o}}\right) Y^{\left(q, r_{o}\right)}[k]\right|^{2} .
$$

\section{Simulations Results}

The simulation results have been obtained using Matlab for the Monte-Carlo results, and using Python 3 with the libraries numpy and gmpy for the computation of the theoretical results (27) and (29) since it requires a high computation precision due to the binomial coefficient. In all simulations, the bandwidth of the LoRa signal is set to $B_{w}=125 \mathrm{kHz}$, the OSR for the new method is $r_{o}=2$, and compared with the usual method at OSR $r_{o}=1^{1}$. Moreover, when a Rayleigh channel is considered, the channel gain is set to $\sigma_{h}^{2}=1$.

\footnotetext{
${ }^{1}$ The code corresponding the theoretical achievable SER at OSR 1 can be found at https://github.com/b-com/ber_LoRa
} 
Fig. 2 shows the SER performance versus SNR (dB) of the LoRa demodulation at OSR 1 for both the even and the odd samples (corresponding to (18) and (23), respectively), compared with the suggested demodulation at OSR 2 (26). In Fig. 2-(a), the AWGN channel is considered, and the SNR varies in the range $[-15,-5] \mathrm{dB}$, and in Fig. 2-(b), the Rayleigh channel is considered, and the SNR varies in the range $[-23,10]$ dB. We observe in both Figs. 2-(a) and (b) that the SER performance of the LoRa demodulation at OSR 1 is the same for both the even and the odd samples. Moreover, a gain of $3 \mathrm{~dB}$ is achieved by the suggested method at OSR 2 compared with OSR 1. This is consistent with the analysis, as we noticed that the ratio $\frac{2 k N|2|^{2}}{(k+1) \sigma^{2}}$ within the exponential in (27) is twice larger than the ratio $\frac{k N|2|^{2}}{(k+1) \sigma^{2}}$ in (8), corresponding to a $3 \mathrm{~dB}$ gain.

Fig. 3 compares the SER performance results versus SNR (dB) of the suggested demodulation obtained through analysis ((27) and (29)) and through simulations for SF 7, 8, and 9, and considering AWGN (a), and Rayleigh (b) channel models. In any configuration, we observe that the theoretical results match the simulations ones, therefore validating the suggested performance analysis. Furthermore, a gain of about $3 \mathrm{~dB}$ is achieve between the performance of "SF N" compared with "SF N-1", which is consistent with the usual demodulation at OSR 1.

\section{CONCLUSION}

In this paper, we have introduced a new demodulation method for oversampled LoRa signal. The principle consists in splitting the received signal into downsampled signals, processing them using dedicated DFT, then combining them and finally applying a usual LoRa demodulation to the combined signals. The advantages of the suggested method lie in the improvement of the demodulation performance compared with the usual one performed at OSR 1, while keeping a low computational cost. Furthermore, a performance analysis in term of SER is developed, which is validated through simulations. Future works will investigate the impact of non-ideal reception including synchronization mismatch on the performance of the demodulation.

\section{REFERENCES}

[1] G. A. Akpakwu, B. J. Silva, G. P. Hancke, and A. M. Abu-Mahfouz, "A Survey on 5G Networks for the Internet of Things: Communication Technologies and Challenges," IEEE Access, vol. 6, no. 12, pp. 3619 - 3647, December 2017.

[2] M. Winkler, "Chirp signals for communications," IEEE WESCON Convention Record, p. 7, 1962.

[3] L. Vangelista, "Frequency Shift Chirp Modulation: The LoRa Modulation," IEEE Signal Processing Letters, vol. 24, no. 12, pp. 1818 - 1821, December 2017.

[4] M. Chiani and A. Elzanaty, "On the LoRa Modulation for IoT: Waveform Properties and Spectral Analysis," IEEE Internet of Things Journal, vol. 6, no. 5, pp. 8463-8470, October 2019.

[5] G. Ferré and A. Giremus, "LoRa Physical Layer Principle and Performance Analysis," proc. of IEEE International Conference on Electronics, Circuits and Systems 2018 (ICECS'18). Bordeaux, France: IEEE, 12 2018, pp. 1 - 4.

[6] T. Elshabrawy and J. Robert, "Closed-Form Approximation of LoRa Modulation BER Performance," IEEE Communications Letters, vol. 22, no. 9, pp. 1778 - 1781, September 2018.

[7] J. Proakis and M. Salehi, Digital Communications. McGraw-Hill, 2008.

[8] C. F. Dias, E. R. de Lima, and G. Fraidenraich, "Bit Error Rate Closed-Form Expressions for LoRa Systems under Nakagami and Rice Fading Channels," Sensors, vol. 19, no. 20, pp. 1 - 11, October 2019. 


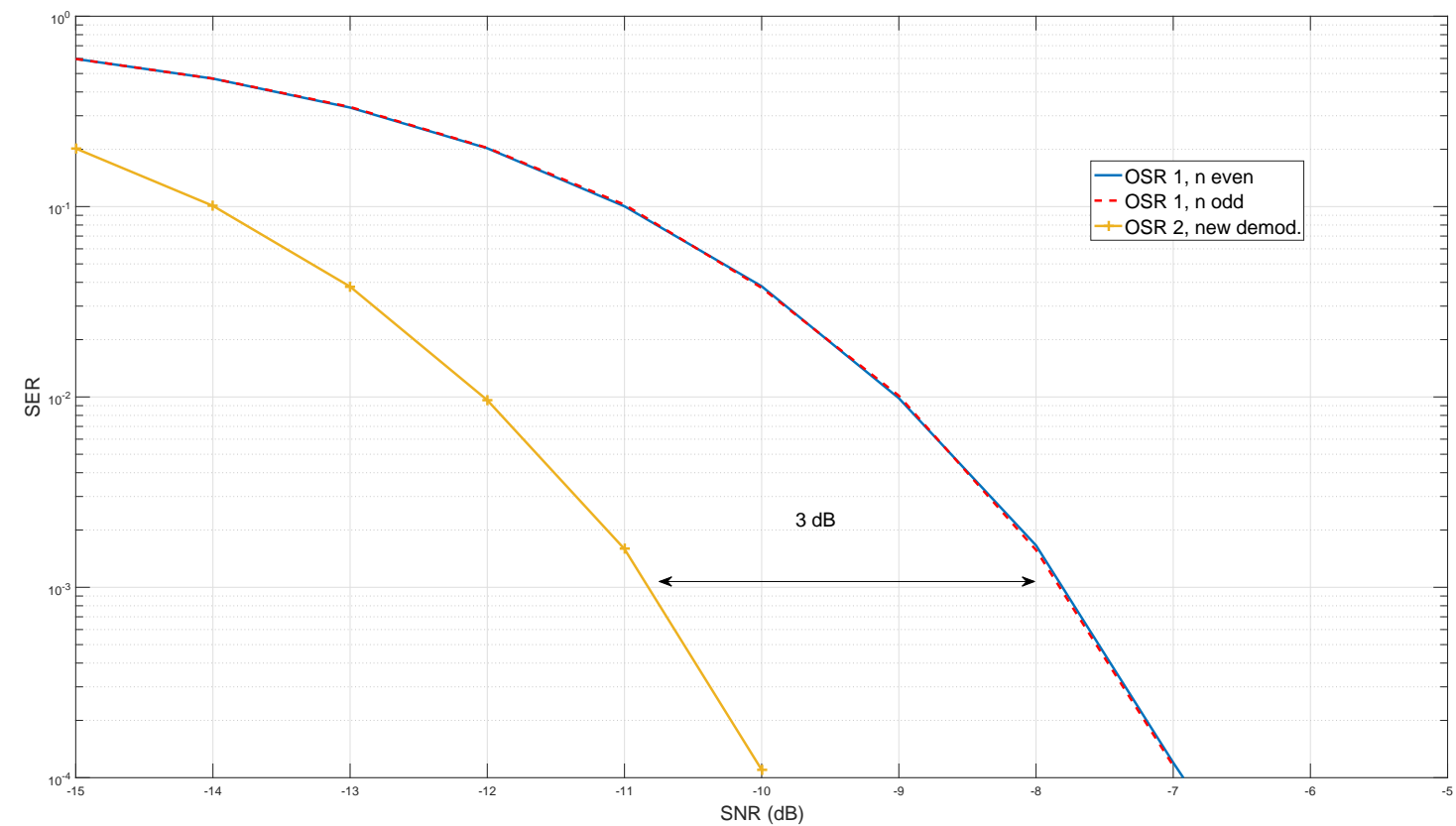

(a) AWGN channel.

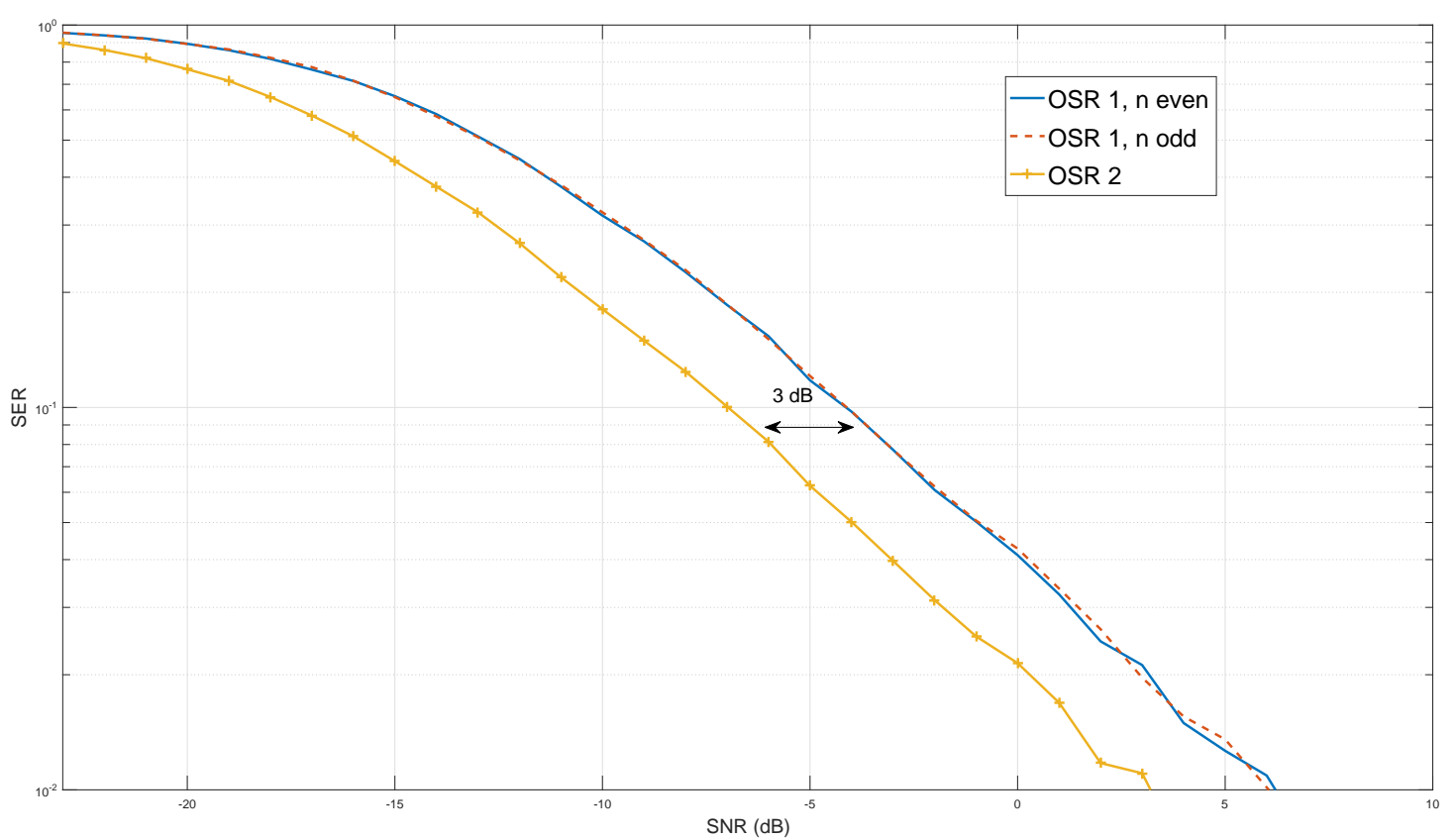

(b) Rayleigh channel.

Fig. 2. SER versus SNR (dB) of the usual LoRa demodulation at OSR1, compared with the suggested new demodulation at OSR2. 


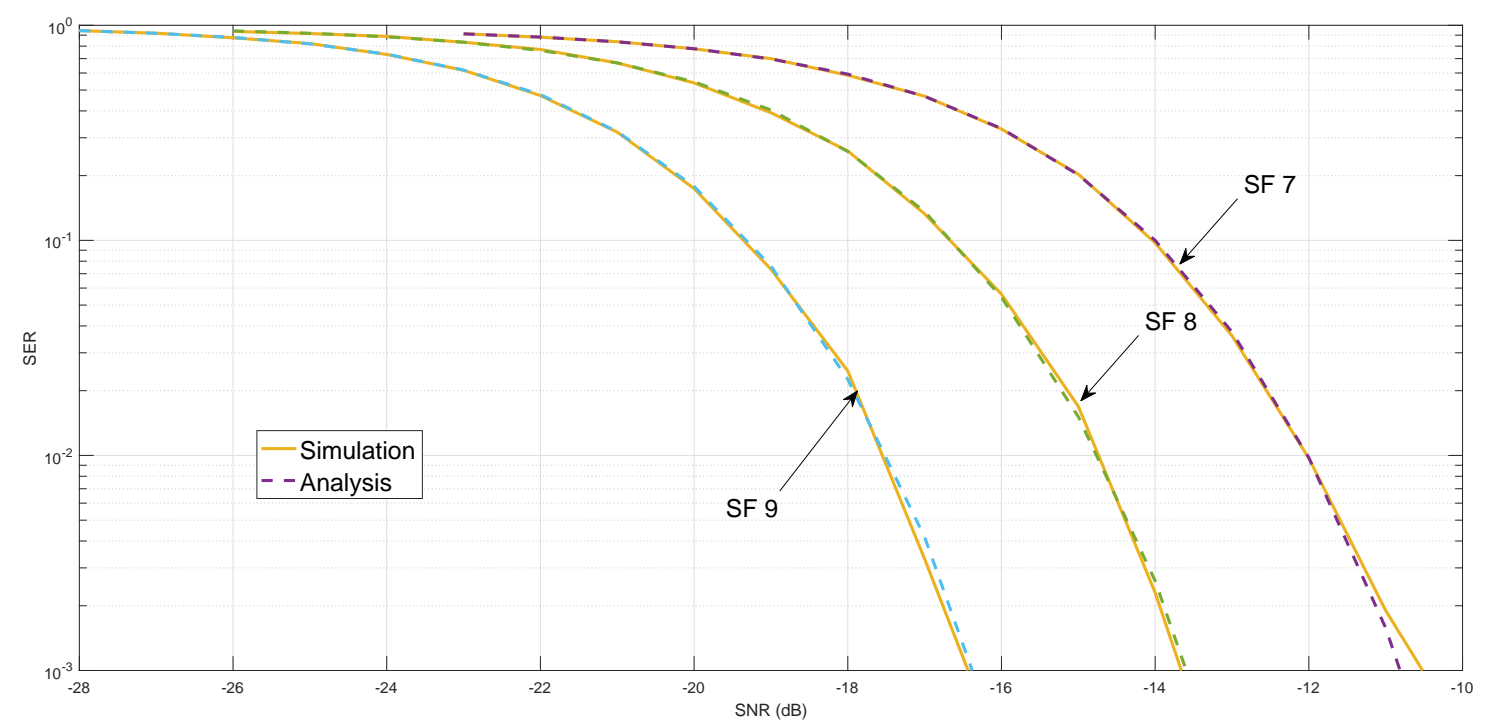

(a) AWGN channel.

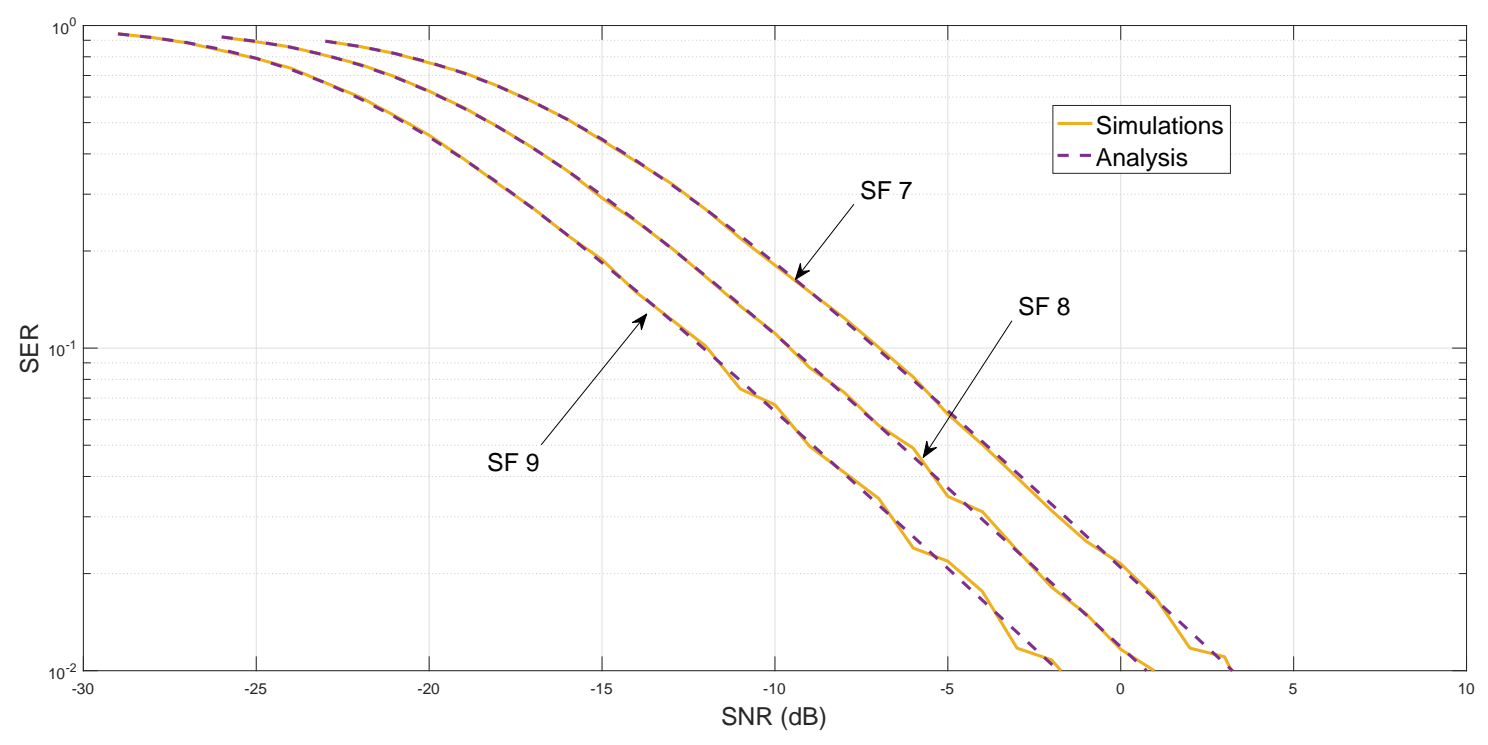

(b) Rayleigh channel.

Fig. 3. SER versus SNR (dB) of new demodulation at OSR2. Comparison between simulations and analytical results for SF 7,8 , and 9 .

[9] V. Savaux and G. Ferré, "Simple Asymptotic BER Expressions for LoRa System over Rice and Rayleigh Channels." San Fransisco, CA, USA: proc. of WTS'21, April 2021, pp. 1 - 5.

[10] V. Savaux, "An Improved Demodulation for LoRa Through a Threshold-Based Detection of Error," TechRxiv, 82021. [Online]. Available: https://www.techrxiv.org/articles/preprint/An_Improved_Demodulation_for_LoRa_Through_a_Threshold-Based_ Detection_of_Error/14877078

[11] M. Xhonneux, D. Bol, and J. Louveaux, “A Low-complexity Synchronization Scheme for LoRa End Nodes," ArXiv, no. arXiv:1912.11344, pp. 1 - 10, December 2019.

[12] R. Ghanaatian, O. Afisiadis, M. Cotting, and A. Burg, "Lora Digital Receiver Analysis and Implementation." Brighton, United Kingdom: proc. of ICASSP'19, May 2019, pp. 1498-1502. 
[13] C. Bernier, F. Dehmas, and N. Deparis, "Low Complexity LoRa Frame Synchronization for Ultra-Low Power Software-Defined Radios," IEEE Transactions on Communications, vol. 68, no. 5, pp. 3140-3152, May 2020.

[14] A. Marquet and N. Montavont, “Carrier and Symbol Synchronisation for LoRa Receivers.” Lyon, France: proc. of EWSN'20, February 2020, pp. $1-6$. 